\title{
EXPERIMENTAL INCISION OF THE INTERVERTEBRAL DISC
}

\author{
J. W. Smith and R. Walmsley, St Andrews, Scotland \\ From the Bute Department of Anatomy, University of St Andrews
}

Interest in the results of injury to the intervertebral disc in man may be said to have had its origin in the work of the Dresden school of pathologists under Schmorl, some of whose findings were incorporated in a report by Beadle (1931). It is well established that one of the lesions that may affect the intervertebral disc is a rupture of the annulus fibrosus with a consequent prolapse of the nucleus pulposus, and that if this prolapse is directed towards the vertebral canal or the intervertebral foramina it may cause pressure on the spinal cord or the nerve roots. The correlation of this pathology with radicular and cord symptoms, and the introduction of operative treatment by Mixter and Barr (1934), have afforded many opportunities of studying the lesion at operation. However, from its very nature, the lesion in man is seldom seen, by the pathologist or surgeon, except in the fully developed stage. It is for this reason that there are few recorded observations either on the structural changes that occur in the disc before an obvious herniation of the nucleus, or on the reparative processes which may occur in the intervertebral disc after the prolapse. With the intention of investigating these problems, a series of operations has been performed on animals in which the annulus fibrosus was incised to a depth sufficient to allow the escape of the nucleus pulposus, and the subsequent macroscopic and microscopic changes in the region of the disc have been observed.

Previous literature-Few accounts of the reaction of the intervertebral disc to operative experimental trauma have been found in the literature. Lob (1933) observed the naked eye and radiological appearances of the vertebrae and disc of rabbits after injury to the annulus fibrosus and found them similar to the changes in spondylosis deformans in man. Filippi (1935), who also worked on rabbits, divided the whole width of the ventral part of the annulus fibrosus. He described a regeneration of the annulus by a proliferation of fibrocartilage from each epiphysial plate, the fibres from the two sides eventually fusing to reconstitute the annulus about forty days after operation. He stated that after a hundred days the damaged disc appeared normal on superficial examination, but the site of the nucleus pulposus was found in microscopic section to be occupied by fibrocartilaginous tissue in which the lamellae extended dorso-ventrally. More recently Key and Ford (1948) published their observations on fourteen dogs in which they had incised the dorsal part of the annulus. In contrast to Filippi they found that only the superficial part of the wound healed, there being a complete failure of healing in the deeper part. In a discussion that followed a communication of these observations, Compere stated that in monkeys on which he had made experimental lesions of the annulus involving loss of the nucleus, arthritic changes developed at the margins of adjacent vertebrae. Thus the evidence must be regarded as incomplete and in some respects contradictory.

\section{THE INTERVERTEBRAL DISC IN THE RABBIT}

The investigation was necessarily carried out on animals in which the intervertebral disc is large enough to permit an operative incision to be made in it with precision, and small enough to allow microscopic sections of it and the adjacent vertebrae to be prepared without undue distortion of the tissues. The rabbit was found to satisfy these conditions.

For the purposes of description the intervertebral disc is considered to have cranial and caudal surfaces and a peripheral surface that has ventral, dorsal and lateral aspects. On the cranial and caudal surfaces of the disc in young rabbits are the thin plate-like epiphyses of 


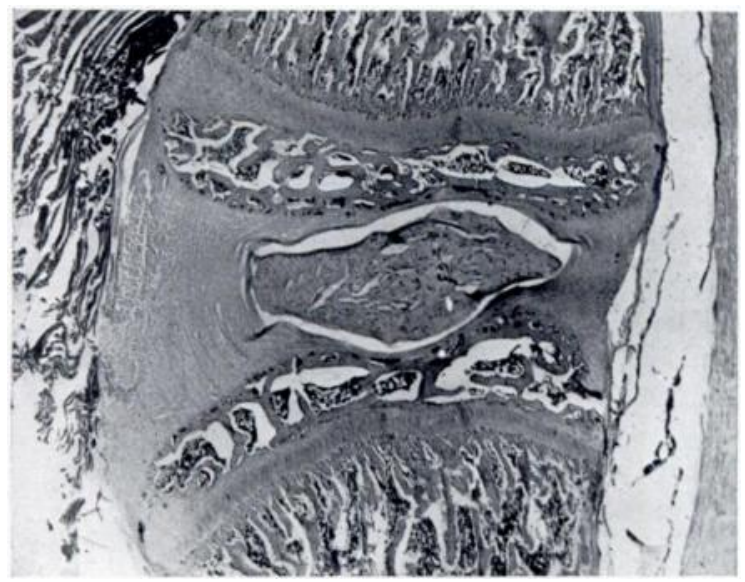

FIG. 1

Normal intervertebral disc and parts of adjacent vertebrae of young rabbit $(\times 11)$. This and all succeeding photomicrographs are taken from sagittal sections which are orientated so that the ventral surface of the disc is directed towards the left-hand side of the page.

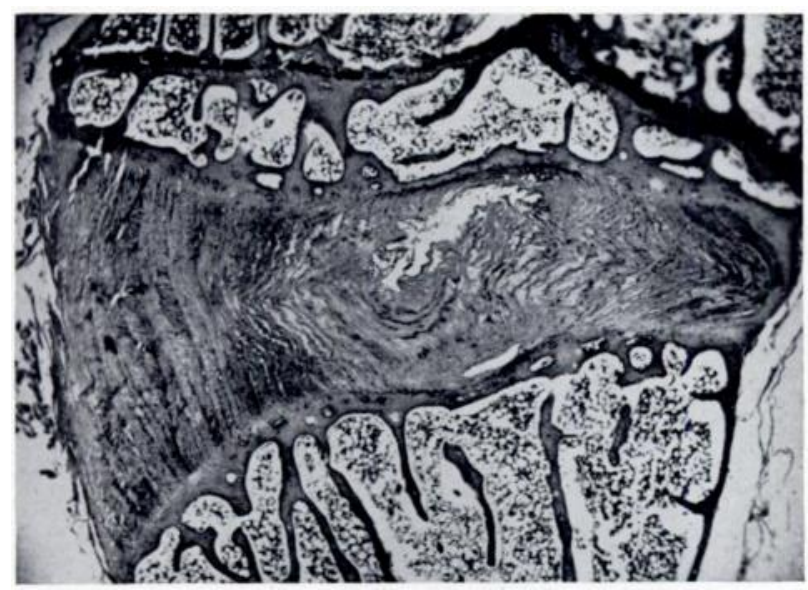

FIG. 2

Normal intervertebral disc and parts of adjacent vertebrae of rabbit aged thirty months $(\times 10)$.

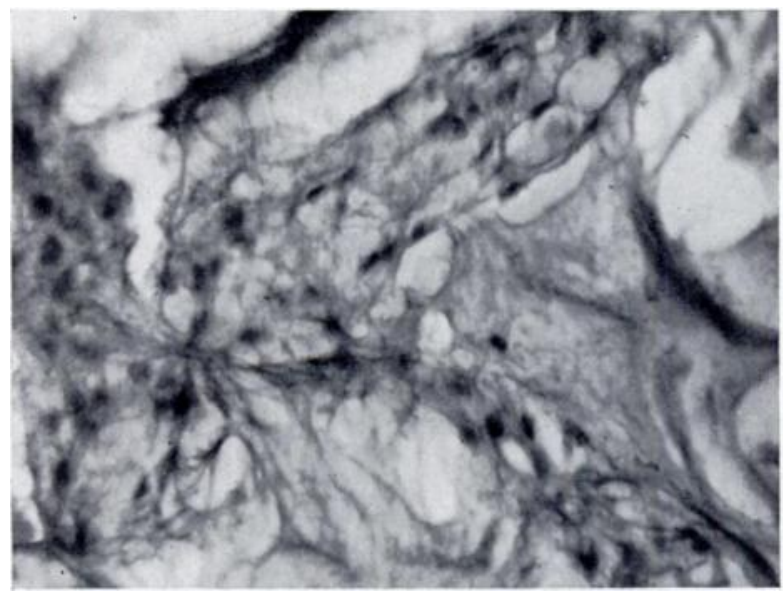

Fig. 3

" Notochordal" cells and fibrous stroma of nucleus pulposus of young rabbit. The cell on the left can be seen to extend from the upper to the lower edge of the photomicrograph $(\times 300)$.

VOL. 33 B, No. 4, NOVEMBER 1951 
the vertebral bodies (Fig. 1), and it is noteworthy that this is the form of epiphysis found in pronogrades generally in contrast to the annular type found in man. The caudal epiphysis fuses with the vertebral body at about two and a half years and the cranial epiphysis soon after (Fig. 2). The annulus fibrosus is formed of concentric lamellae of fibrocartilage, disposed obliquely, and since adjacent lamellae have different obliquities the annulus has, on section, a striated appearance which becomes increasingly apparent with age. The nucleus pulposus in the lumbar region lies nearer to the dorsal than to the ventral surface of the disc; in most sections there is a space between the annulus and the nucleus which is regarded as an artefact caused by shrinkage of the nucleus during preparation of the sections.

The structure of the nucleus varies with age, and these alterations are analogous to the age changes in man described by Keyes and Compere (1932). In young rabbits the nucleus is sharply demarcated from the surrounding annulus. The matrix consists of a structureless, basophilic mucoid material traversed by loose wavy fibres and showing in sections large vacuoles. Embedded in the matrix are a few fibroblasts and cartilage cells but the predominant cellular element is large multinuclear cells with acidophilic protoplasm (Fig. 3). These cells, which may be regarded as multinuclear syncytia, are similar to the cells found in the nucleus of the young child and have been considered by Keyes and Compere (1932) to be derivatives of the notochord; they will be referred to subsequently as "notochordal" cells. With increase in age the demarcation of the nucleus from the annulus becomes less abrupt, the notochordal cells disappear, and the nuclear matrix becomes denser and is infiltrated with fibroblasts and cartilage cells which appear to proliferate from the annulus (Fig. 2).

In these experiments immature rabbits were used; the epiphyses of the vertebral bodies were not fused and the predominant cells in the nucleus pulposus were the multinuclear derivatives of the notochord.

The avascular nature of intervertebral discs appears to be well established and confirmation of this was obtained by injecting India ink in plasma into the aortae of several rabbits immediately after death. The injection was made at an average pressure of 100 millimetres mercury and maintained for upwards of six hours. The typical appearance of thick sections of a disc from such an animal is shown in Figures 4 to 6, and the general vascular pattern of the vertebral epiphysis and surrounding tissue is apparent. The vessels of the diaphysis and epiphysis of the body of the vertebrae can be seen but the disc itself is avascular except for a few small vessels in its peripheral part. In this feature, which is of great importance in the present problem of reaction to injury, the rabbit's disc resembles that of man, and it is postulated that both have a low rate of metabolism and are nourished by diffusion of fluids from surrounding tissues and especially from the epiphyses of the vertebral bodies.

It can be said therefore that the rabbit's disc resembles the human disc in general structure. Nevertheless, it was accepted at the outset of the investigation that any conclusions that might be reached could not, for a number of reasons, be held to be directly applicable to man. For example, the mechanical stresses to which the disc in the rabbit is subjected differ from those which affect the human disc; and also, the rabbit discs, possibly because of the immaturity of the animals, did not show the degenerative changes which have been described as preceding prolapse of the human nucleus pulposus (see Friberg 1948, and Lindblom and Hultquist 1950). It was believed, however, that the results would provide useful data for a similar proposed investigation in monkeys, which, it is considered, will give information which might be more justly applicable to the human disc.

\section{THE OPERATION}

Seventy rabbits were used in the investigation and in each of them a lumbar intervertebral disc was damaged by making a transverse incision in the annulus fibrosus. In all operations the anaesthetic was intravenous nembutal. 


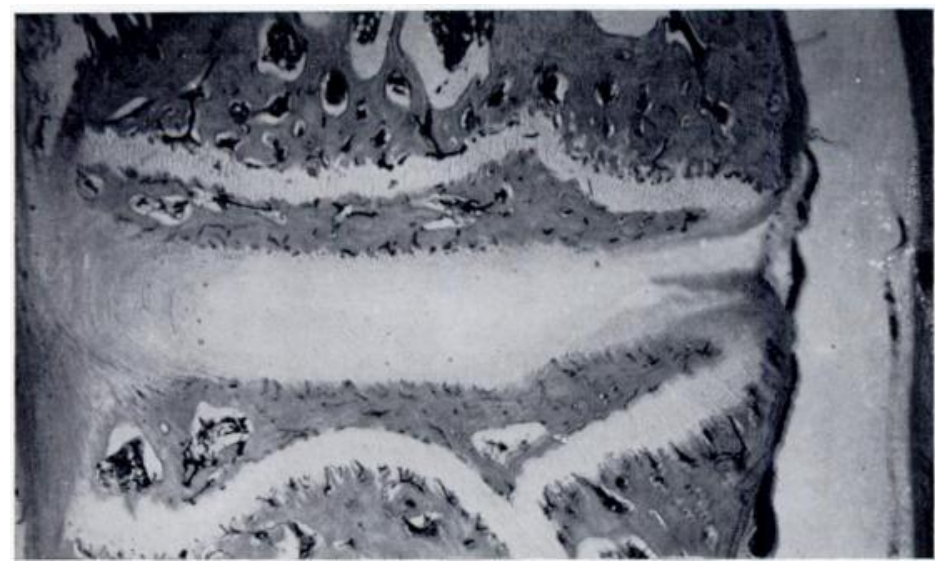

FIG. 4

Thick section $(75 \mu)$ of disc and vertebrae after injection of vessels with India ink. The injected vessels are represented by the black lines and dots, which are absent from the disc $(\times 14)$.

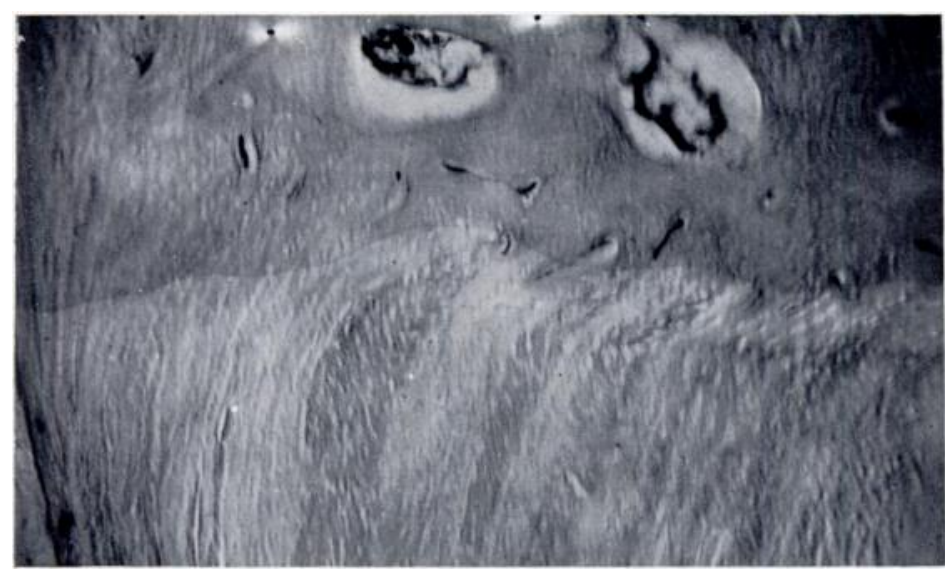

FIG. 5

Ventral part of epiphysis and adjacent part of annulus fibrosus. Two small vessels are seen in the most superficial fibres of the annulus on left of photomicrograph $(\times 60)$.

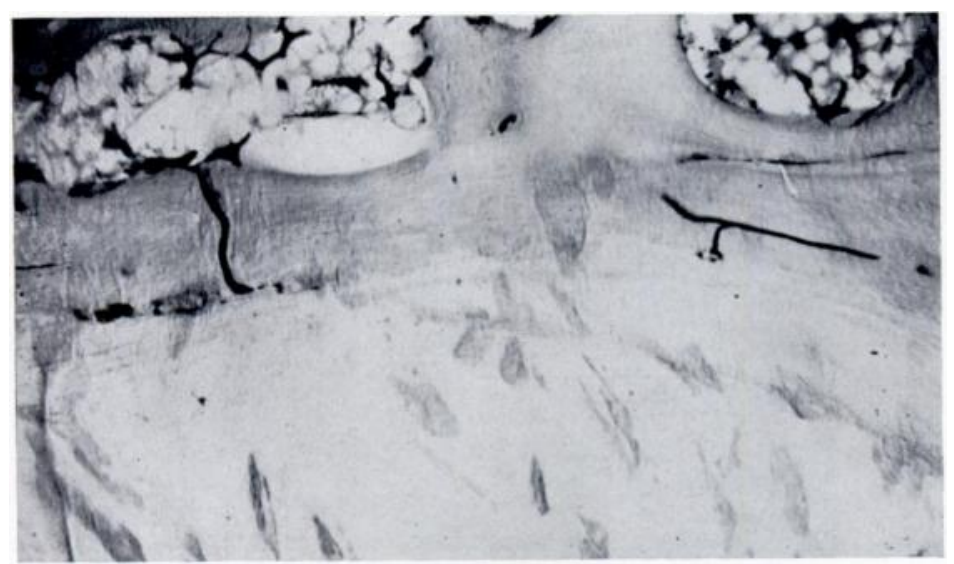

FIG. 6

Central part of epiphysis and adjacent part of nucleus puplosus $(\times 100)$. 
An attempt was made to reach the dorsal aspect of the disc in four rabbits, but this approach proved impracticable because of the large size of the spinal cord relative to the size of the vertebral canal, and because of the rich plexus of epidural veins. The rabbits in which this approach was used showed evidence of cord lesions on recovery from the anaesthetic and were destroyed.

In the remaining sixty-six rabbits the ventral aspect of the disc was incised. A median incision was made in the ventral abdominal wall and carried through the parietal layer of peritoneum. The bowel was displaced to the right, and the abdominal aorta and posterior rena cava were exposed in the median groove between the psoas muscles. The peritoneum was incised on the left side of the aorta, cranial to the posterior mesenteric artery, and when the aorta and vena cava were in turn gently retracted to the right the lumbar arteries and veins of the region became visible. On separation of the psoas muscles midway between

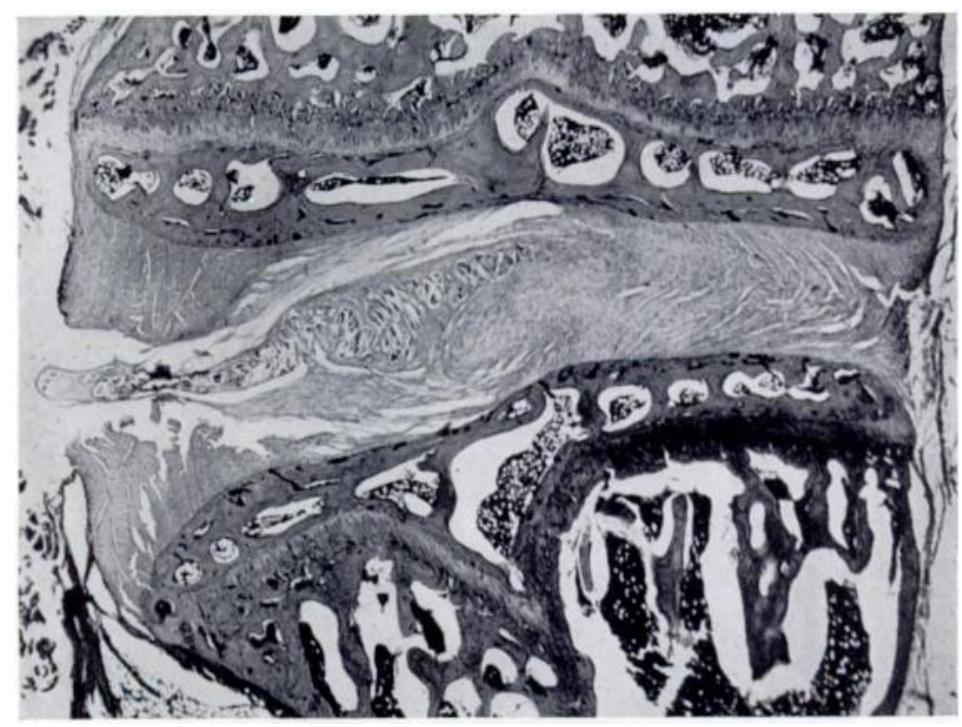

FIG. 7

Intervertebral disc immediately after operation. The ventral part of the annulus fibrosus has been cut transversely and part of the nucleus pulposus lies between the cut fibres $(-14)$.

two adjacent groups of lumbar vessels the glistening intervertebral disc was exposed. The ventral part of the annulus fibrosus, which is about 3 millimetres deep in well grown young rabbits, was then incised transversely, the incision being about 4 millimetres long. In the majority of rabbits the nucleus pulposus immediately prolapsed through the incision and formed a gelatinous bead about the size of a match head on the cut surface of the disc; in a few rabbits, although the incision proved on subsequent section to be into the nucleus, no such bead formation was seen. The abdominal structures were then allowed to settle into place and the abdomen was closed.

Seven rabbits died during or soon after operation and four later showed signs of infection and were destroyed; the remaining fifty-five provided the material on which the observations were made.

\section{OBSERVATIONS}

The disc immediately after operation-The appearance of the disc immediately after operation is shown in Figure 7 . The ventral fibres of the annulus are cut midway between the adjacent vertebrae, and nuclear material is seen to protrude into the incision as a tongue-like process. The material that escapes from the site of the nucleus consists of 


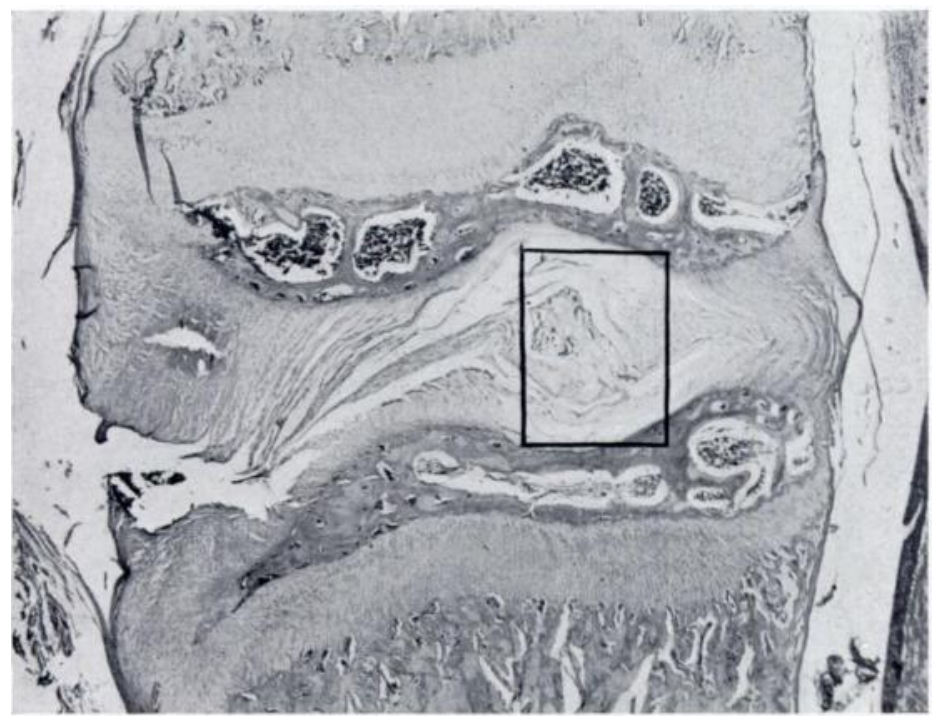

Fig. 8

Intervertebral disc seventeen days after operation. The photomicrograph is from a section near the median plane and there is no evidence of healing in the annulus fibrosus $(\times 14)$.

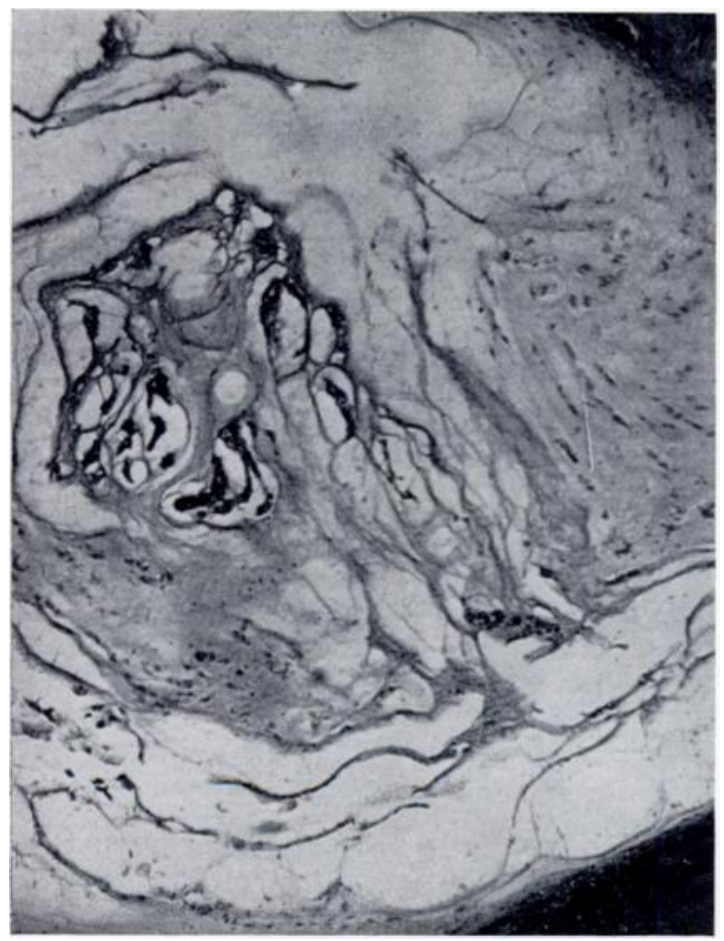

FIG. 9

The enclosed area in Figure 8 showing the nests of " notochordal" cells $(\times 70)$. 
" notochordal cells" embedded in mucoid material; the fibrous element of the nucleus, consisting of fine collagenous fibres and a few fibroblasts and cartilage cells, remains in situ. The escape of the "notochordal" element of the nucleus may not be complete. Figures 8 and 9 show a "notochordal" nest seventeen days after operation while the wound is still unhealed, and in Figure 14 similar collections of cells, now encapsulated, can be seen after seven weeks.

Subsequent changes - The changes which occur in the disc after operation are described under three headings: 1) Healing of the superficial ventral fibres of the annulus; 2) changes in the deep ventral annular fibres; and 3) changes in the nuclear tissue which remains in situ. Healing of the superficial fibres-During the three weeks after the operation the superficial part of the wound in the annulus heals, the proliferative reaction occurring in the fibrous tissue deep to the ventral longitudinal ligament. The healing process begins in the lateral

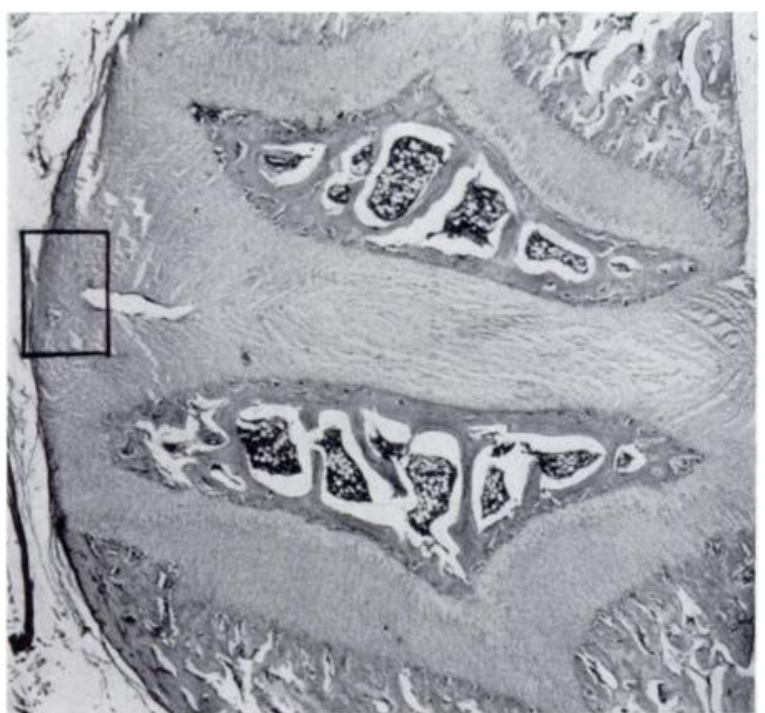

FIG. 10

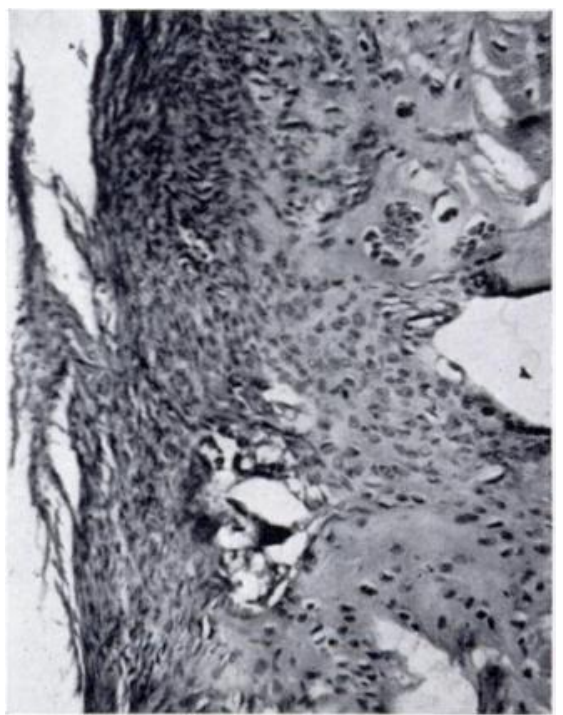

FIG. 11

Figure 10-Section through lateral part of wound ten days after operation $(\times 14)$. Figure $11-$ The enclosed area in Figure 10 showing intense fibroblast reaction associated with the healing of the lateral part of the wound $(\times 105)$

parts of the wound and is well established ten days after operation (Figs. 10 and 11 ). Thereafter, healing spreads towards the median part of the wound but the time taken for closure to be completed varies and is no doubt influenced by the size of the original incision and other factors individual to the animal. Thus Figure 8 , which is from a section near the median plane, shows no evidence of the healing reaction seventeen days after operation whereas in another animal killed nineteen days after operation serial sections, of which Figures 12 and 13 are from a relevant specimen, showed the wound to be completely closed. In most cases complete closure occurs between three and four weeks after operation.

While the closure of the wound is being effected by an active fibrous tissue reaction, a series of changes occurs in the fibrocartilaginous tissue of the annulus immediately surrounding the superficial part of the incision. The first of these changes is a proliferation of the cartilage cells in an oval zone around the superficial part of the incision (Fig. 13), a multiplication which rapidly extends centripetally and centrifugally. This proliferation soon causes a protuberance on the ventral aspect of the intervertebral disc (Figs. 14 and 15) and eventually extends to the ventral surfaces of the adjacent vertebrae and, in young animals, blends with the epiphysial cartilage. As chondrification progresses, and before its spread is complete, an 
oval zone of cartilage in the ventral part of the disc becomes calcified (Fig. 15), and this calcification extends both centripetally and centrifugally so that the entire chondrified zone eventually passes through a calcified phase.

Before calcification is complete, however, ossification occurs in the calcified zone so that

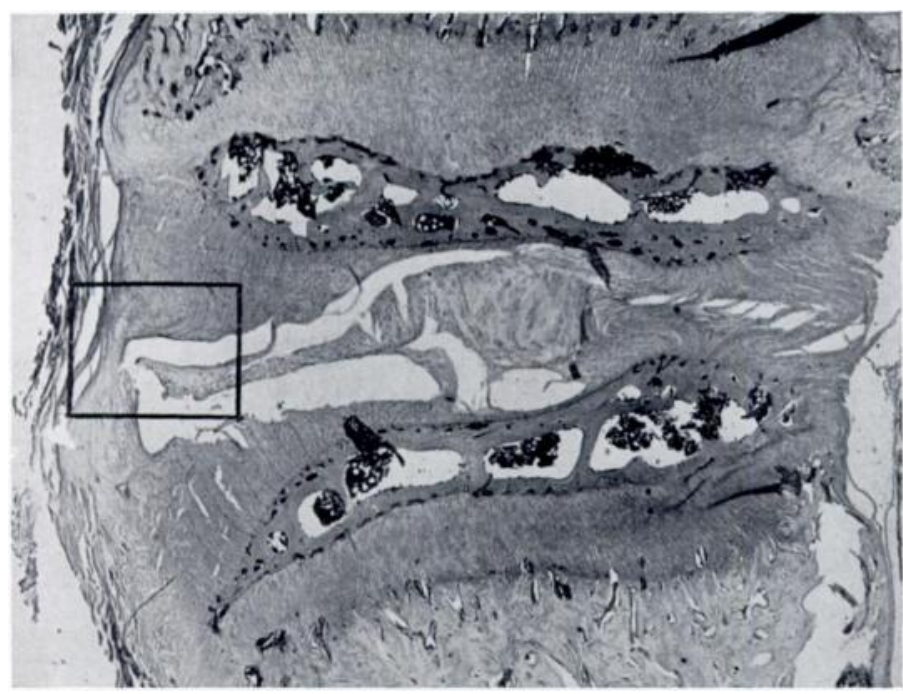

FIG. 12

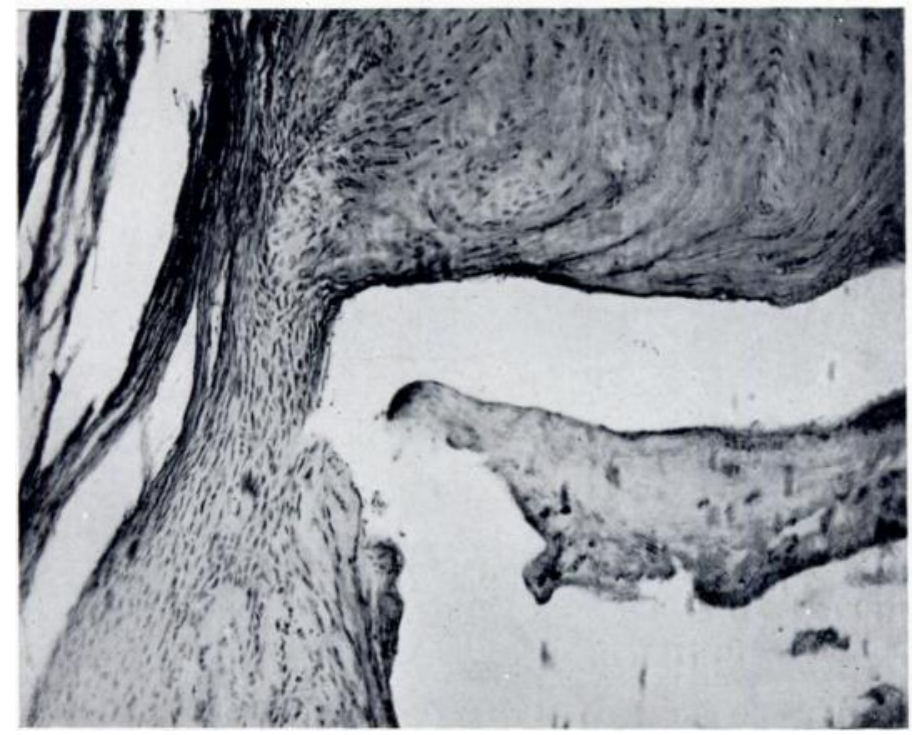

FIG. 13

Figure 12-Section through wound near the median plane nineteen days after operation $(\times 12)$. Figure $13-$ The enclosed area in Figure 12. The healed superficial part of the wound is seen and there is a proliferation of cartilage cells in the cranial lip of the wound $(\times 70)$.

a bony annulet is formed in the ventral part of the disc surrounding the site of the original incision. Like other post-operative changes in the disc, the time of appearance of ossification showed considerable variation, but the earliest evidence of it was observed ninety-one days after operation (Figs. 16 and 17). By the end of the first year, the spread of ossification from the original annulet has completely, or almost completely, replaced the cartilage, a bony 


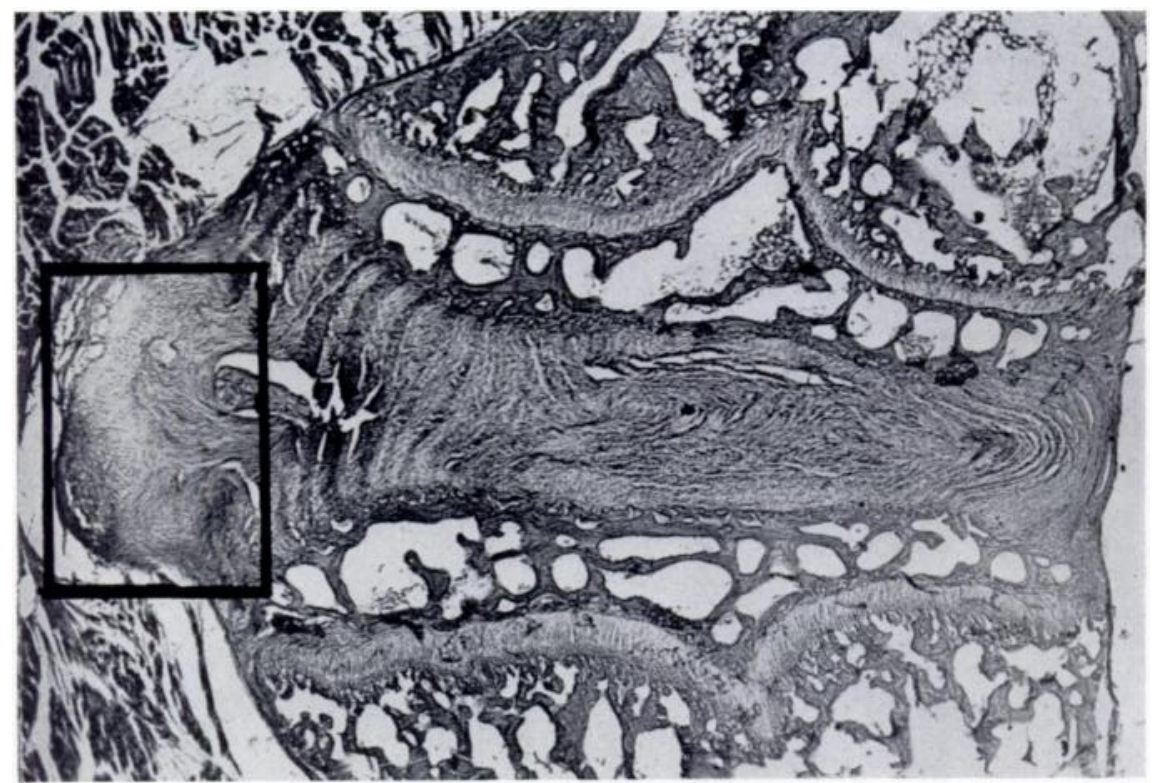

Fig. 14

Section through lateral part of wound seven weeks after operation. As the ring of partly calcified cartilage is cut tangentially near its lateral end only one area of chondrification is seen in this photomicrograph $(\times 16)$.

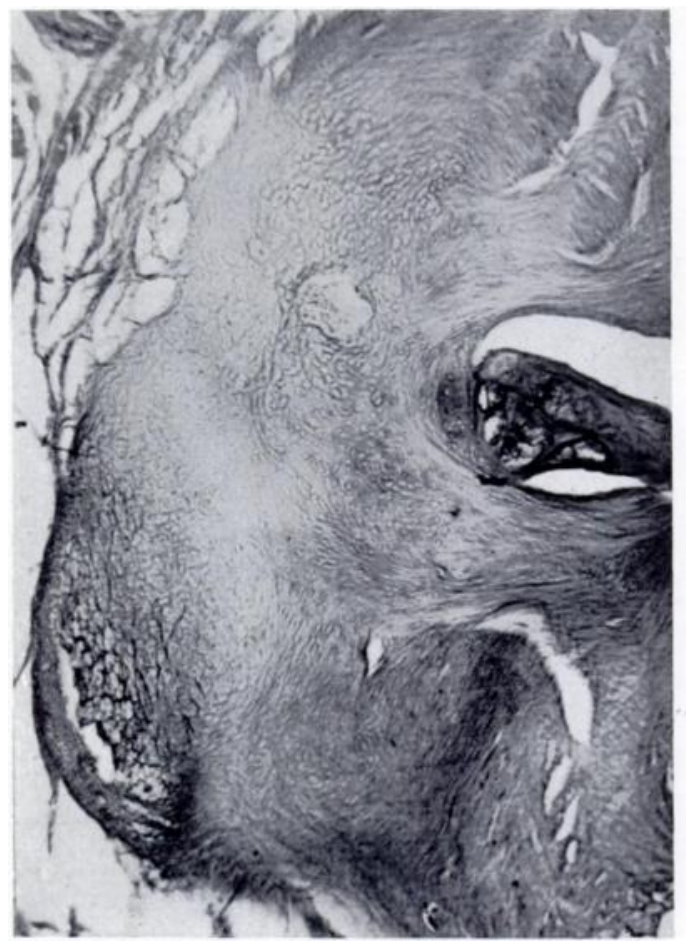

FIG. 15

The enclosed area in Figure $14(\times 40)$. 
ankylosis between the vertebrae adjacent to the damaged disc is established and a considerable bony prominence is formed on the ventral aspect of the disc. The appearance of such an ankylosis is illustrated in Figure 18, which is from a rabbit thirteen months after operation. During the second year after operation the bone constituting the ankylosing bridge does not show any appreciable growth, and Figure 19, which is from a rabbit twenty-five months after operation, is considered to be typical of the appearance found any time after the first year. Changes in the deep ventral annular fibres - The changes that have already been described involve only the more superficial fibres of the ventral part of the annulus fibrosus. The deeper part of the annulus is characterised by its inertness, so that even when bony ankylosis is established superficially the incision in it may still be evident. The prolonged persistence of the wound appears to be closely related to the presence in it of an extension of the tissue from the original site of the nucleus pulposus. This is illustrated in Figure 20 which is taken

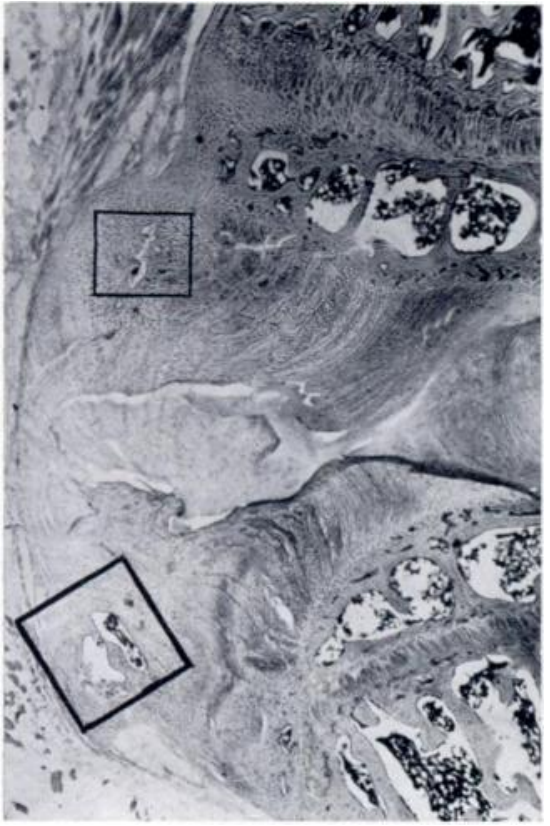

FIG. 16

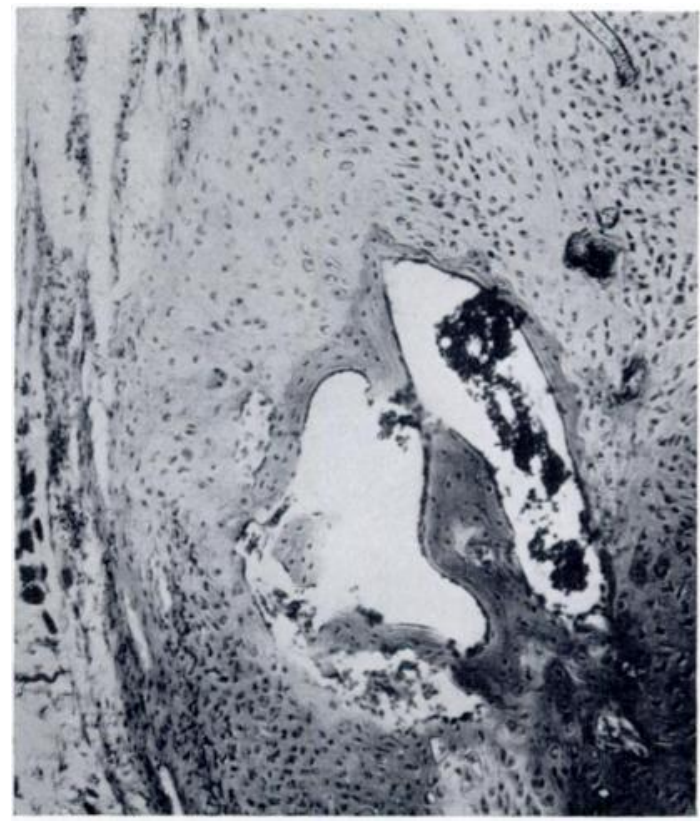

FIG. 17

Figure 16-Ventral part of intervertebral disc thirteen weeks after operation. There is a centre of ossification in the ventral part of the annulus both cranial and caudal to the original wound; these are enclosed within ink squares $(\times 17)$. Figure 17-The caudal (lower) enclosed area in Figure 16 showing commencement of ossification in annulus $(\because 130)$.

from a specimen twenty-one months after operation. Such extensions from the nuclear site do not occupy the entire transverse width of the wound but are limited to its median part. In the lateral parts of the wound where no such tongue-like extension is present, the deep part of the wound is gradually healed during the first year by a slow appositional spread in a medial direction of the chondrification already noted in the superficial lamina of the lateral part of the annulus. It is for this reason that there is an absence of the original wound in Figures 18 and 19, both of which photomicrographs are taken from sections lateral to the mid-line.

Changes in the nuclear tissue which remain in situ-When the annulus fibrosus is incised most of the mucoid element of the nucleus pulposus passes through the wound and carries with it the majority of the " notochordal " cells. The fibrous element of the nucleus remains in situ, and lying among its collagenous fibres are fibroblasts and a few cartilage cells. During the six months after the operation there is a rapid proliferation of both fibroblasts and cartilage

vol. 33 B, No. 4, November 1951 


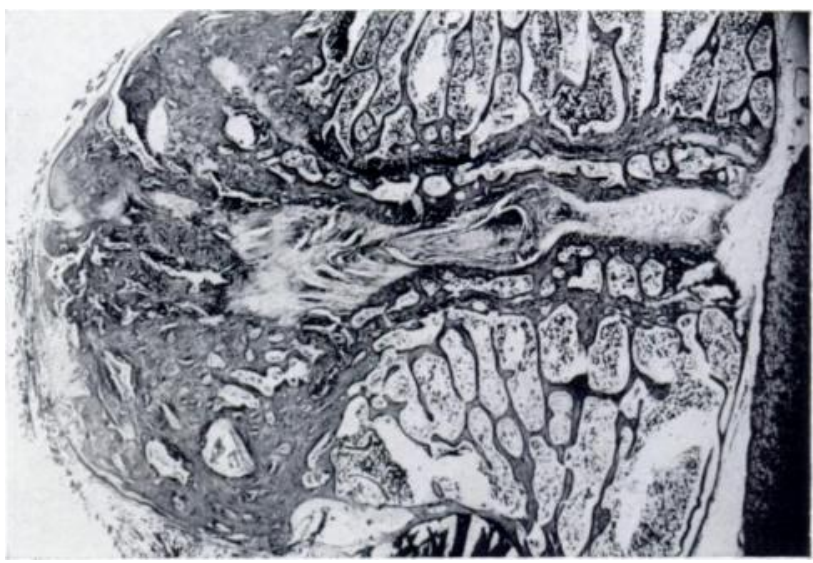

Fig. 18

Section through lateral part of wound thirteen months after operation. There is now a considerable bony prominence on the ventral surface of the disc, ankylosing adjacent vertebrae $(\times 12)$.

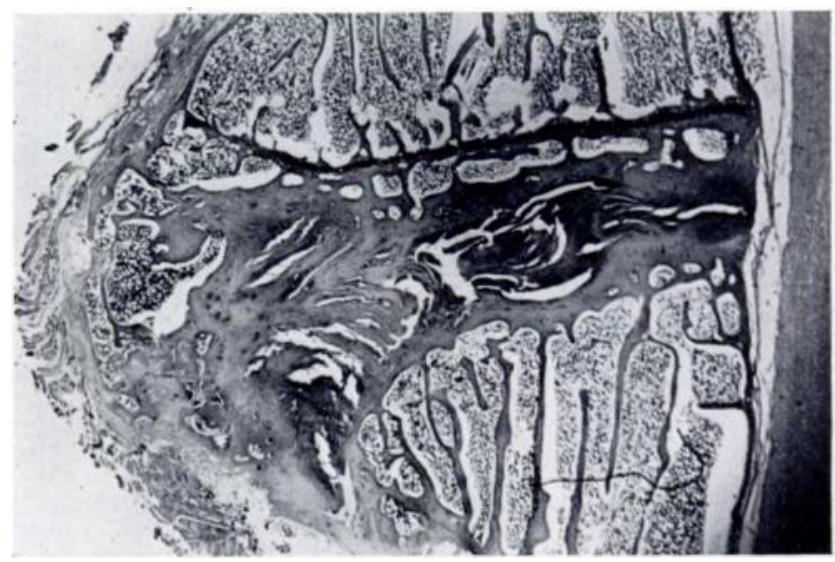

FIG. 19

Section through lateral part of wound twent $y$-five months after operation $(\times 9)$.

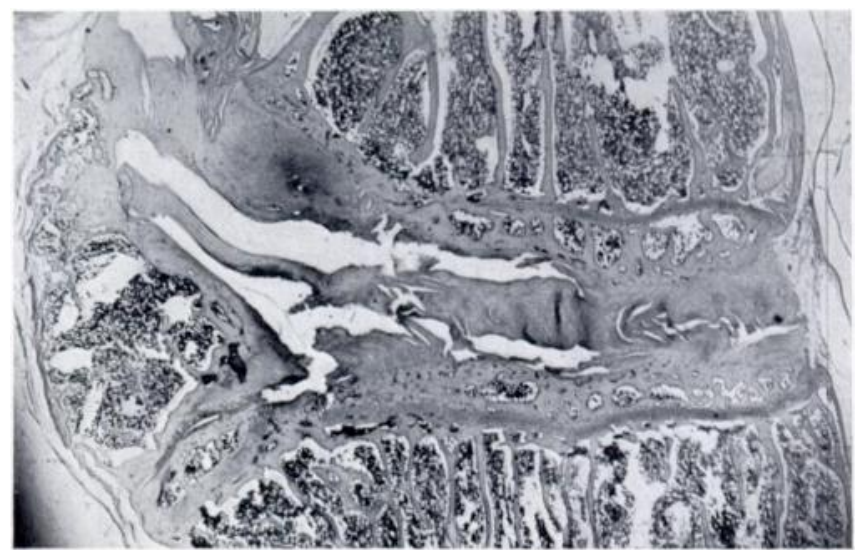

FIG. 20

Section through median part of wound twenty-one months after operation. The original operative incision is present and the tissue in the nuclear site extends into it $(\times 8)$.

THE JOURNAL OF BONE AND JOINT SURGERY 


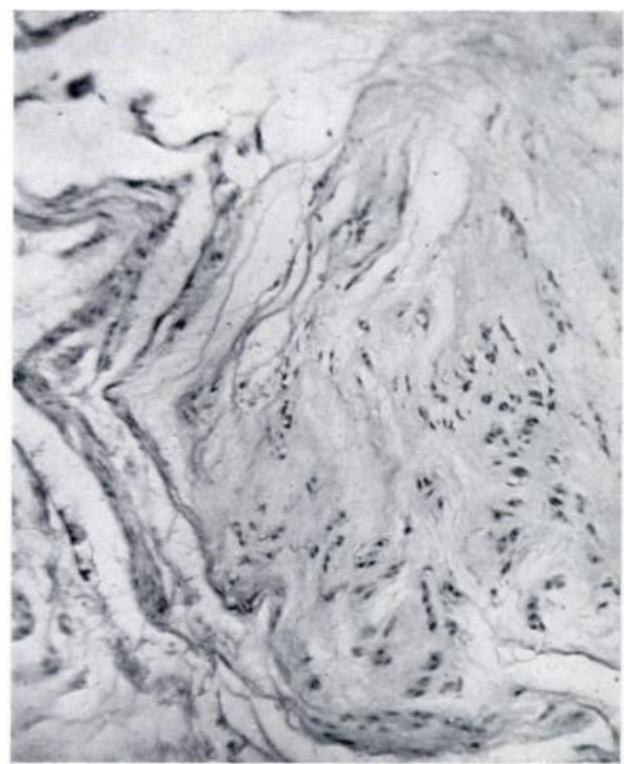

Fig. 21

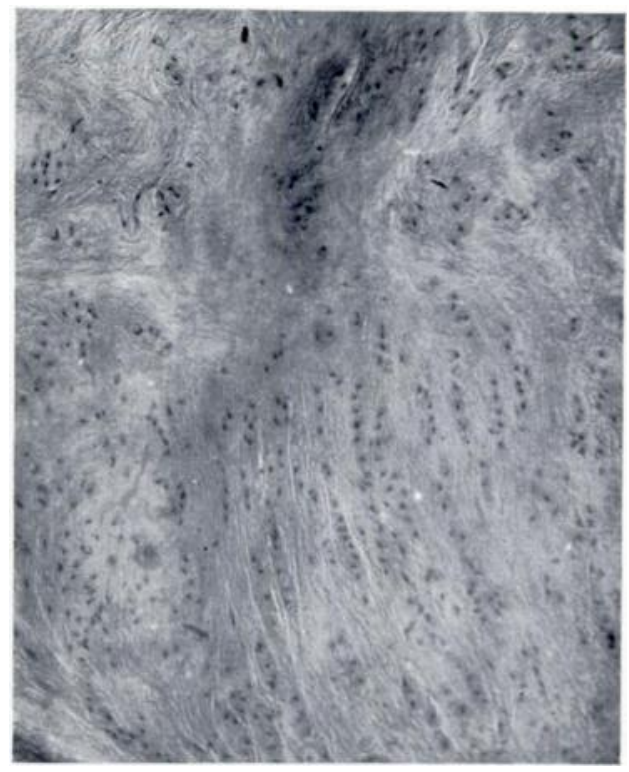

FIG. 22

Figure 21-Tissue in site of nucleus pulposus three weeks after operation $(\times 65)$. Figure 22-Tissue in site of nucleus pulposus nine weeks after operation. The increased density of the tissue is evident and the linear arrangement of the cartilage cells can be seen $(\times 110)$.

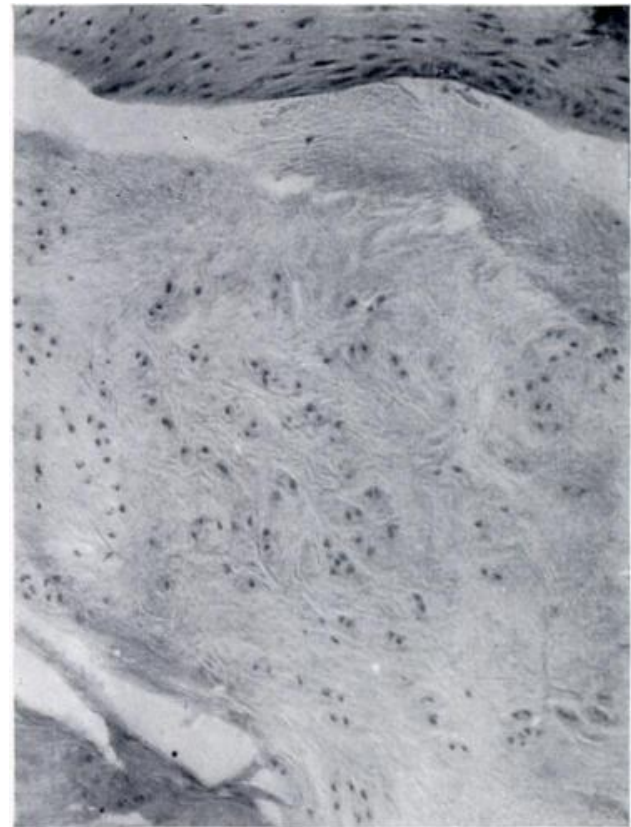

FIG. 23

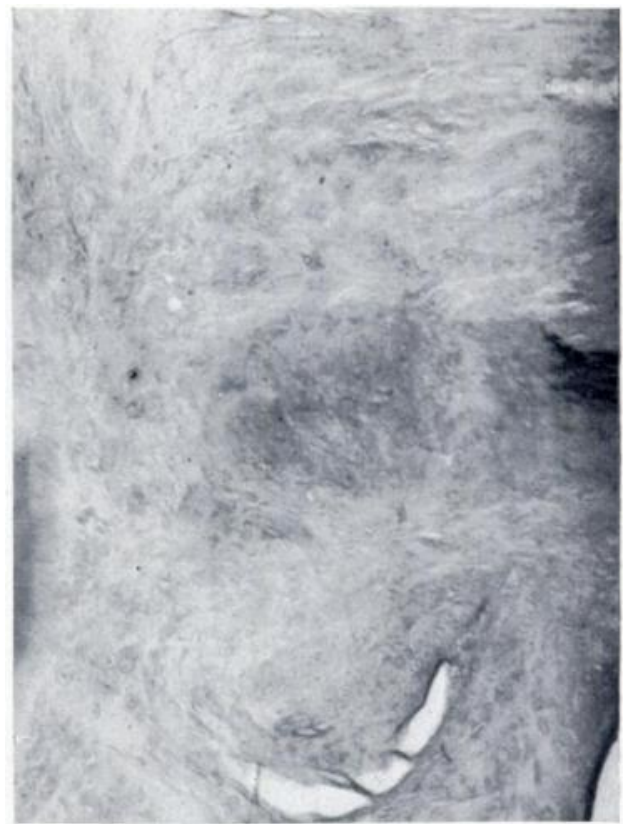

FIG. 24

Figure 23-Extension of tissue from nuclear site into deep part of wound nine weeks after operation. This photomicrograph is from same section as Figure 22 but shows grouped arrangement of cartilage cells $(\times 110)$. Figure 24-Tissue in site of nucleus pulposus twenty-one months after operation. Few cells are present and the collagenous tissue is extremely dense $(\times 100)$. 
cells (Fig. 21 ), and with an increase in collagenous fibres the tissue becomes increasingly dense; this tissue reaction also occurs in the tongue-like process in the deep part of the wound. Throughout the first four months the cartilage cells are especially active; in the original nuclear site they tend to arrange themselves in columns in the line of the long axis of the vertebral column (Fig. 22), whereas in the process that lies between the cut edges of the wound the cells are aggregated in small groups (Fig. 23). During the later part of the first year and throughout the second year after operation the tissue becomes increasingly dense and both the cartilage cells and fibroblasts decrease in number (Fig. 24).

\section{DISGUSSION}

The observations that have been made on fifty-five rabbits during the two vears after an incision in the ventral part of the annulus appear to present a clearly defined sequence of changes. The healing of the superficial part of the wound is correlated with the vascular nature of the prevertebral tissues and the fibroblastic reaction is typical of that observed in tissue healing in general. The failure of healing in the deep part of the wound is in keeping with the experimental findings of Key and Ford (1948) and is associated with the recognised avascularity of the deeper annular fibres; at the same time we consider that the presence in this part of the wound of an extension of the tissue that has proliferated in the site of the

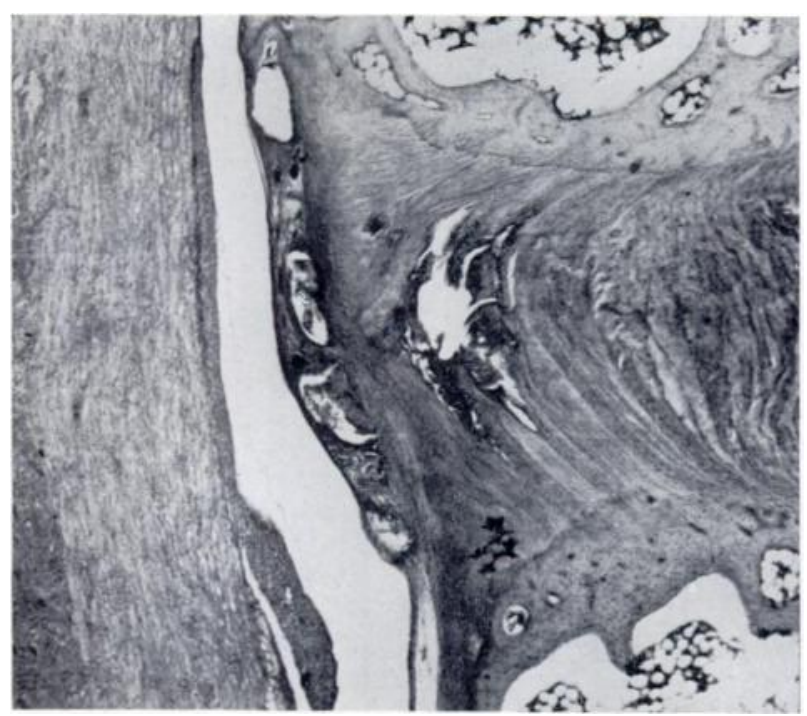

FIG. 25

Dorsal part of intervertebral disc of mature rabbit. In this photomicrograph the dorsal surface of the disc is on the left and a part of the spinal cord and a nerve root are shown. No operation had been performed on this rabbit, but the rupture of several lamellae of the annulus is seen and also several small masses of the nucleus pulposus underlying the dorsal longitudinal ligament $(\times \mathbf{2 0})$. nucleus must be considered an additional factor. The annular fibres which are related to such extensions usually have a "clean-cut" appearance and their free ends assume the form of an encapsulating membrane; several of the illustrations (Figs. 13, 14, 15 and 20) show this feature.

The cartilaginous and bony reactions in the ventral part of the disc have been a constant feature in all rabbits that were kept sufficiently long after operation. Two factors have been considered in the analysis of these changes, the one being the upset in the normal mechanism of the joint, and the other the trauma of the wound. The normal mechanism of the joint is dependent on an intact annulus fibrosus and nucleus pulposus, and it is considered that any gross interference with these structures will lead to a tissue reaction in them which tends to immobilise the joint. The final ossification of the ventral part of the annulus may thus be correlated with the proliferation of collagenous tissue in the original site of the nucleus, the two processes proceeding concurrently and in harmony with each other.

Lamination in the annulus fibrosus is a recognised feature of its structure, and each lamella is a discrete unit whose fibres have a different direction from those that are immediately adjacent to it. It is postulated that the composite form of the annulus is important in the mechanism of rupture of the annulus fibrosus in man. An increase in pressure in the nucleus pulposus is the direct result of any compression force applied to the adjacent vertebrae, and 
this pressure is transmitted in turn to the surrounding annulus fibrosus and most directly to the deepest lamella; tension in all the lamellae may be further increased by movement of the vertebral column. If the stress so produced is great enough, or if the strength of the lamellae has been reduced by degenerative changes, the deepest fibres of the disc may rupture and allow the nucleus to prolapse between their torn ends. Repetition of similar trauma may cause further lamellae, now under more direct pressure, to give way, so that a progressive tracking of the nucleus to the periphery of the disc occurs. Evidence of such fractional rupture of the annulus has been seen in the posterior part of the non-operated intervertebral discs of two rabbits with an accompanying prolapse of the nucleus pulposus into the tear (Fig. 25). It has been observed that the tears in adjacent lamellae are not always opposite one another so that the track may have an irregular course from the nucleus.

The investigation of the relative importance of degenerative changes in the annulus fibrosus and trauma to the disc appears to us to be of primary importance in the elucidation of the etiology of prolapse of the nucleus pulposus.

\section{SUMMARY}

1. The normal anatomy of the intervertebral disc of immature rabbits is described.

2. An account is given of the changes that occur after an operative incision in the ventral part of the intervertebral discs of rabbits which allowed the escape of the nucleus pulposus. The account is based on observations made on fifty-five young animals killed at intervals during the twenty-five months after operation.

3. The superficial part of the wound in the annulus heals rapidly by active fibrosis. Thereafter there is a chondrification of the ventral region of the disc, followed by ossification. A prominent bony ridge ultimately ankyloses the vertebrae adjoining the disc.

4. The site of the nucleus pulposus is eventually occupied by a dense pad of fibrocartilage. A tongue of this tissue projects into the deep median part of the wound which remains unhealed.

5. A hypothesis is submitted regarding the mechanism of rupture of the annulus fibrosus and prolapse of the nucleus pulposus in man; this hypothesis is based in part on the observations of lesions in discs not subjected to operation.

We wish to express our thanks to Mr James Brown of this Department for his technical assistance during the investigation.

\section{REFERENCES}

BeAdle, O. A. (1931): The Intervertebral Discs: Observations on their Normal and Morbid Anatomy in Relation to Certain Spinal Deformities. London: Medical Research Council Special Report, Series No. 161. FilipPI, A. (1935): La guarigone del disco intervertebrale dopo asportazione del nucleus pulposus negli animali da esperimento. Chirurgia degli Organi di Movimento, 20, 1.

Friberg, S. (1948): Anatomical studies on lumbar disc degeneration. Acta Orthopaedica Scandinavica, 17, 224.

Key, J. A., and Ford, L. T. (1948): Experimental intervertebral disc lesions. Journal of Bone and Joint Surgery, 30-A, 621 .

Keyes, D. C., and Compere, E. L. (1932): The normal and pathological physiology of the nucleus pulposus of the intervertebral disc: an anatomical, clinical and experimental study. Journal of Bone and Joint Surgery, 14, 897.

Lindblom, K., and Hultquist, G. (1950): Absorption of protruded disc tissue. Journal of Bone and Joint Surgery, 32-A, 557.

LoB, A. (1933): Die Zusammenhänge zwischen den Verletzungen der Bandscheiben und der Spondylosis deformans im Tierversuch. Deutsche Zeitschrift für Chirurgie, 240, 421.

MiXter, W. J., and BARR, J. S. (1934): Rupture of intervertebral disc with involvement of spinal canal. New England Journal of Medicine, 211, 210.

vol. 33 B, No. 4, NOVEmber 1951 